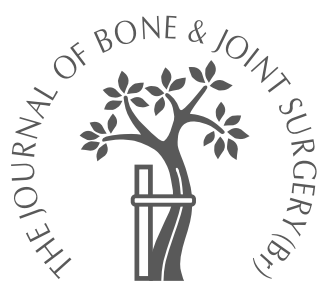

R. Bhattacharya, U. T. Vassan, P. Finn, A. Port

From James Cook University Hospital, Middlesbrough, England

\title{
Sanders classification of fractures of the os calcis
}

\author{
AN ANALYSIS OF INTER- AND INTRA-OBSERVER VARIABILITY
}

Our study was undertaken to assess the inter- and intra-observer variability of the classification system of Sanders for calcaneal fractures. Five consultant orthopaedic surgeons with different subspecialty interests classified CT scans of 28 calcaneal fractures using this classification system. After six months, they reclassified the scans.

Kappa statistics were used to analyse the two groups. The interobserver variability of the classification system was 0.32 (95\% confidence interval (CI) 0.26 to 0.38 ). The subclasses were then combined and assessment of agreement between the general classes as a whole gave a kappa value of $0.33(95 \% \mathrm{Cl} 0.25$ to 0.41$)$. The mean kappa value for intra-observer variability of the classification system was $0.42(95 \% \mathrm{Cl} 0.22$ to 0.62$)$. When the subclasses were combined, it was $0.45(95 \% \mathrm{Cl} 0.21$ to 0.65$)$.

Our results show that, despite its popularity, the classification system of Sanders has only fair agreement among users.

The os calcis is the tarsal bone which is most frequently fractured. ${ }^{1}$ However, despite extensive clinical experience of this injury, the final outcomes remain poor. Displaced intra-articular fractures of the calcaneum have always been difficult to treat. A recently published randomised, controlled trial, comparing operative and non-operative treatment of calcaneal fractures, has shown a better outcome in some groups of surgically-treated patients, ${ }^{2}$ but controversy persists. The surgeon's inability to classify these injuries consistently has been blamed as one of the reasons for the difficulty in treating them. ${ }^{3}$ To overcome this problem, various classification systems have been proposed for intra-articular fractures of the calcaneum based either on plain radiography ${ }^{4,5}$ or $\mathrm{CT}^{6-9}{ }^{6}$ Sanders ${ }^{9}$ described his classification system as the logical extension of that of Souer and Remy ${ }^{5}$ which was based on the number of articular fragments seen on radiography. ${ }^{9}$ The system of Sanders ${ }^{3}$ has been found to be useful in determining treatment as well as prognosis. ${ }^{3}$

It is based on the coronal CT scan of the calcaneal fracture which shows the widest undersurface of the posterior facet of the talus. ${ }^{9}$ The calcaneum is divided into three columns by two fracture lines A and B (Fig. 1). A third fracture line $\mathrm{C}$, separates the sustentacular fragment from the posterior facet of the calcaneum, thereby giving rise to four potential articular fragments. Type 1 is an undisplaced fracture irrespective of the number of fragments and type 4 is a severely comminuted fracture usually with four or more parts. Type 2 is essentially a two-part fracture akin to a longitudinal split of the tibial plateau, and is subdivided into A, B or C, depending on the position of the primary fracture line. Type 3 is similarly a three-part fracture with a central depressed fragment akin to a split-depression fracture of the tibial plateau and is again subdivided into $\mathrm{AB}, \mathrm{BC}$ or $\mathrm{AC}$, depending on the presence of a combination of two fracture lines.

Despite the popularity of the classification system, and like so many other classification systems for fractures in other parts of the body, there has been no measurement of its reproducibility until the study of Furey et $\mathrm{al}^{10}$ who determined interobserver reliability. However, in their study, the classes and subclasses were assumed to be ordinal. We felt that although this may be justified in regard to the classes themselves, the assumption of the subclasses as ordinal (i.e. $2 \mathrm{~A}$ is better than $2 \mathrm{~B}$, or $3 \mathrm{AC}$ is better than $3 \mathrm{BC}$ ), may be challenged. We also decided that it was imperative to check the intra-observer reliability of this system.

\section{Patients and Methods}

We studied the CT scans of 28 patients with calcaneal fractures who presented over the last year in our department. Five consultant ortho- 


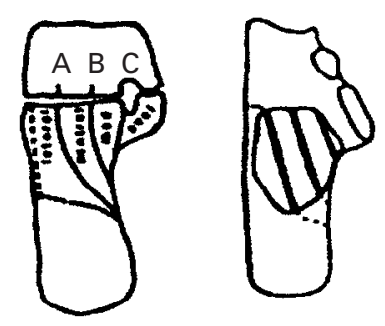

Type I

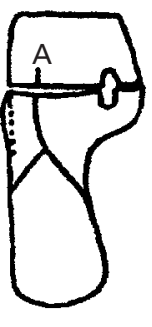

Type IIA
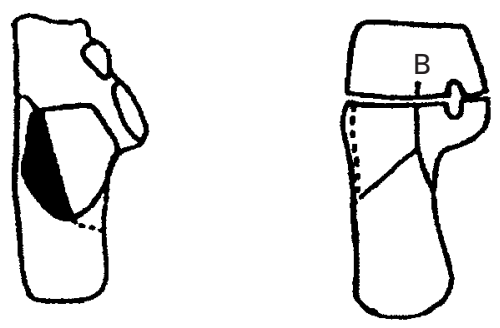

Type IIB
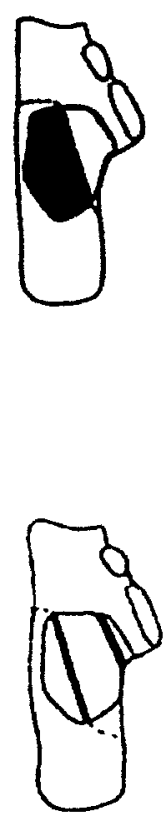

Type III AC
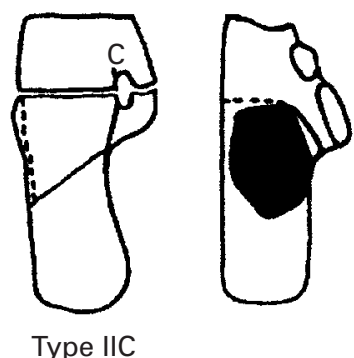

Type IIC

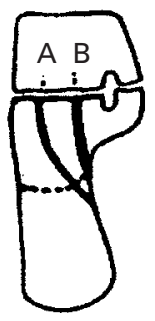

Type III AB
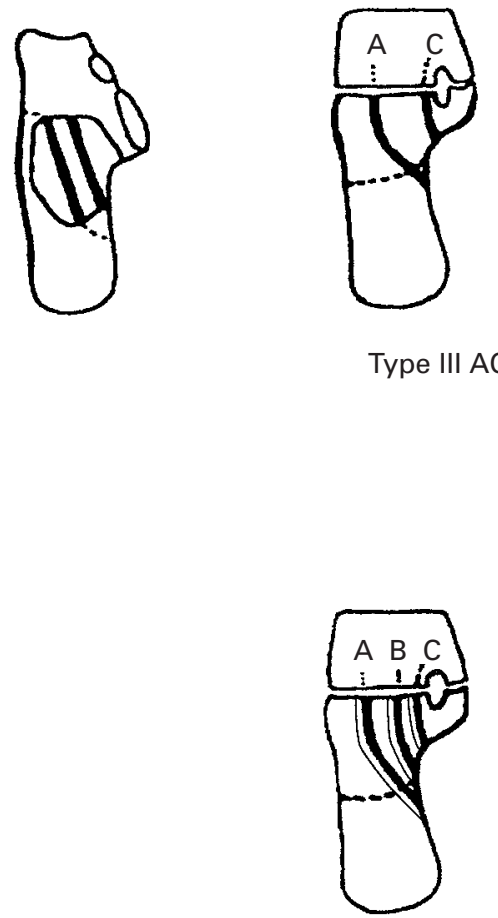

Type IV

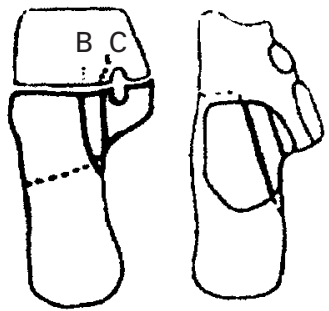

Type III BC

Fig. 1

The classification system of Sanders ${ }^{13}$ for fractures of the os calcis (reproduced with permission from the Journal of Orthopaedic Trauma). 
Table I. Details of the interpretation of Kappa values according to Landis and $\mathrm{Koch}^{12}$

\begin{tabular}{ll}
\hline Kappa value & Agreement \\
\hline$<0.0$ & Poor \\
0.00 to 0.20 & Slight \\
0.21 to 0.40 & Fair \\
0.41 to 0.60 & Moderate \\
0.61 to 0.80 & Substantial \\
0.81 to 1.00 & Almost perfect \\
\hline
\end{tabular}

Table II. Intra-observer Kappa values among the five surgeons

\begin{tabular}{lll}
\hline Surgeon subspecialty & With subtypes & Classes as a whole \\
\hline Foot and ankle & 0.62 & 0.65 \\
Lower limb arthroplasty & 0.47 & 0.57 \\
Limb reconstruction & 0.37 & 0.46 \\
Upper limb & 0.36 & 0.36 \\
Knee & 0.22 & 0.21 \\
\hline
\end{tabular}

Table III. Total number of CT scans classified according to type based on the classification of Sanders ${ }^{13}$ according to individual observer, by number and percentage

\begin{tabular}{lrrrrrrrr}
\hline Observer & \multicolumn{2}{c}{ Type I } & \multicolumn{2}{c}{ Type II } & \multicolumn{2}{c}{ Type III } & \multicolumn{2}{c}{ Type IV } \\
\hline A1 & 3 & 10.7 & 10 & 35.7 & 12 & 42.9 & 3 & 10.7 \\
A2 & 9 & 32.1 & 11 & 39.3 & 6 & 21.4 & 2 & 7.1 \\
B1 & 1 & 3.6 & 12 & 42.9 & 11 & 39.3 & 4 & 14.3 \\
B2 & 1 & 3.6 & 12 & 42.9 & 9 & 32.1 & 6 & 21.4 \\
C1 & 0 & 0.0 & 6 & 21.4 & 16 & 57.2 & 6 & 21.4 \\
C2 & 1 & 3.6 & 7 & 25.0 & 16 & 57.1 & 4 & 14.3 \\
D1 & 2 & 7.1 & 17 & 60.7 & 8 & 28.6 & 1 & 3.6 \\
D2 & 2 & 7.1 & 13 & 46.5 & 10 & 35.7 & 3 & 10.7 \\
E1 & 1 & 3.6 & 8 & 28.6 & 11 & 39.3 & 8 & 28.6 \\
E2 & 0 & 0.0 & 7 & 25.0 & 14 & 50.0 & 7 & 25.0 \\
\hline
\end{tabular}

paedic surgeons were specifically chosen to represent as wide a range of subspecialty interests as possible. Apart from one 'foot and ankle' specialist, the other subspecialties included arthroplasty, limb reconstruction, the upper limb and the knee. All the surgeons regularly dealt with trauma and hence saw calcaneal fractures in their routine clinical practice. The identification labels of all the scans were removed. The complete set of CT scans were presented to each consultant and he was asked to classify the fractures using the system of Sanders. A description along with a diagrammatic explanation of the classification system from a standard textbook was also supplied to each consultant. Each was also asked to choose his mode of treatment for each fracture (i.e. conservative or operative). After a mean interval of six months, the same scans were given to the same five consultants, along with the description and diagrammatic explanation, and they were asked to reclassify the fractures using the same system.

The two sets of classifications from each consultant were then tabulated and Kappa statistics were used to analyse the inter- and intra-observer variations. The Kappa is a coefficient of agreement and has values ranging from +1 representing complete agreement, to zero representing agreement no better than chance alone, to -1 representing complete disagreement. There is no universally agreed level of acceptability for Kappa values which have thus been interpreted in various ways. One interpretation in common use is that described by Fleiss ${ }^{11}$ in which a Kappa value of 0.4 indicates poor agreement, that between 0.4 and 0.75 fair agreement and that above 0.75 strong agreement. Another slightly more lenient interpretation is that by Landis and $\operatorname{Koch}^{12}$ (Table I). The Kappa statistics were first calculated for the classification system including the subclasses. The subclasses were then combined and further Kappa values were calculated to assess agreement between the general classes as a whole. The $95 \%$ confidence intervals $(\mathrm{CI})$ were then calculated for all the values.

\section{Results}

The detailed results of the five surgeons are shown in Tables II to IV. The results for interobserver reliability showed a Kappa value of 0.32 with a $95 \%$ confidence interval (CI) of 0.26 to 0.38 . When the subclasses were combined and an assessment made of the interobserver agreement between the general classes as a whole, the Kappa value was 0.33 with a $95 \%$ CI of 0.25 to 0.41 . The tests for intra-observer variability for the classification system including the subclasses gave a mean Kappa value of 0.42 with a range of 0.22 to 0.62 . When the subclasses were combined, the mean Kappa value for the intra-observer variability of the

Table IV. Total number of CT scans classified according to subtypes based on classification of Sanders ${ }^{13}$ according to individual observer

\begin{tabular}{lllllllll}
\hline Observer & Type I & Type IIA & Type IIB & Type IIC & Type IIIAB & Type IIIAC & Type IIIBC & Type IV \\
\hline A1 & 3 & 10 & 0 & 0 & 8 & 3 & 1 & 3 \\
A2 & 9 & 10 & 1 & 0 & 5 & 0 & 1 & 2 \\
B1 & 1 & 7 & 5 & 0 & 7 & 3 & 1 & 4 \\
B2 & 1 & 8 & 3 & 1 & 7 & 1 & 1 & 6 \\
C1 & 0 & 5 & 1 & 0 & 9 & 6 & 1 & 6 \\
C2 & 1 & 6 & 1 & 0 & 9 & 6 & 1 & 4 \\
D1 & 2 & 11 & 6 & 0 & 7 & 0 & 1 & 1 \\
D2 & 2 & 12 & 1 & 0 & 7 & 0 & 3 & 3 \\
E1 & 1 & 8 & 0 & 0 & 7 & 3 & 1 & 8 \\
E2 & 0 & 4 & 2 & 1 & 9 & 5 & 0 & 7
\end{tabular}


general classes as a whole was 0.45 with a range of 0.21 to 0.65 .

These results showed that the interobserver agreement of the classification system as a whole was poor to fair at best and only slightly better when the subclasses were combined. The intraobserver reliability was mostly poor to moderate with only one surgeon obtaining substantial agreement between his two readings. The results also showed that although the foot and ankle specialist scored the highest for consistency, there was no real correlation between the specialty interest and consistency in using the Sanders classification system.

A statistical analysis was also performed to assess the variability in the management decisions of the 28 fractures between the various surgeons. A Cochran $Q$ test was used for the analysis showing that there was no significant difference $(Q=1.63 ; \mathrm{p}$ value $=0.44)$ among the surgeons with regard to the management of the fractures.

\section{Discussion}

One of the essential requirements of a classification system is that it should be reproducible so that it can help in the assessment of data. There is a consistent, universal agreement regarding the options for treatment of intra-articular fractures of the calcaneum based on the classification of Sanders, according to which, as the fracture line moves from the lateral to medial, the operative view becomes progressively poor and the ability to obtain an accurate reduction of the fracture more difficult. ${ }^{9}$ This forms the basis for the prognostic value of this classification system, which has been shown to have a good correlation between the types of fracture and outcome. ${ }^{9}$

Only if there is consistency within observers and between observers can a valid conclusion be drawn about the reliability of a classification system. Our results do not match the conclusions reached by Furey et $\mathrm{al}^{10}$ who found the interobserver variation to be 0.56 (0.32 in our study) for the classification system as a whole and 0.48 (0.33 in our study) when the subclasses were combined. This could partly be explained by the fact that our study had more observers than that of Furey et al. ${ }^{10}$ This difference in the values between the two studies itself emphasises the variability of the system. Additionally, our study shows that intra-observer reliability varies widely among different orthopaedic surgeons which defeats the purpose of a classification system. However, irrespective of the classification of the fracture, there appeared to be a consensus on the management of the fractures, suggesting that, although required for prognostic purposes, a meticulous classification of the fractures may not be essential for correct management decisions, contrary to the views of Sanders. ${ }^{3}$

We conclude that, despite the popularity of the classification system of Sanders for intra-articular fractures of the calcaneum, there is a high degree of variability and inconsistency in its interpretation with only a fair to moderate agreement among its users. Our aim is neither to advocate this system nor to undermine it, but to highlight its shortcomings so that caution is exercised in its usage and interpretation.

We would sincerely like to thank the following consultant orthopaedic surgeons at the James Cook University Hospital, Middlesbrough, for kindly sparing their time to classify the CT scans: Mr A. C. Hui, Mr I. McMurty, Mr R. J. Montgomery and Mr A. Rangan.

No benefits in any form have been received or will be received from a commercial party related directly or indirectly to the subject of this article.

\section{References}

1. Koval KJ, Zuckerman JD. Handbook of fractures. Second ed. London: Lippincott Williams and Wilkins, 2002:261.

2. Buckley R, Tough S, McCormack R, et al. Operative compared with nonoperative treatment of displaced intra-articular calcaneal fractures. J Bone Joint Surg [Am] 2002;84-A:1733-44.

3. Sanders R. Displaced intra-articular fractures of the calcaneus. J Bone Joint Surg [Am] 2000;82-A:225-50.

4. Essex-Lopresti P. The mechanism, reduction technique, and results in fractures of the os calcis. Br $J$ Surg 1952;39:395-419.

5. Soeur R, Remy R. Fractures of the calcaneus with displacement of the thalamic portion. J Bone Joint Surg [Br] 1975;57-B:413-21.

6. Zwipp H, Tscherne H, Thermann H, Weber T. Osteosynthesis of displaced intraarticular fractures of the calcaneus: results in 123 cases. Clin Orthop 1993;290:76-86.

7. Crosby LA, Fitzgibbons TC. Computerized tomography scanning of acute intraarticular fractures of the calcaneus: a new classification system. J Bone Joint Surg [Am] 1990;72-A:852-9.

8. Eastwood DM, Gregg PJ, Atkins RM. Intra-articular fractures of the calcaneum. Part 1: pathological anatomy and classification. J Bone Joint Surg [Br]1993;75-B:183-8.

9. Sanders R, Fortin P, Dipasquale T, Walling A. Operative treatment in 120 displaced intra-articular calcaneal fractures: results using a prognostic computed tomography scan classification. Clin Orthop 1993;290:87-95.

10. Furey A, Stone C, Squire D, Harnett J. Os calcis fractures: analysis of interobserver variability in using Sanders classification. J Foot Ankle Surg 2003;42:21-3.

11. Fleiss JL. Statistical methods for rates and proportions. Second ed. New York: John Wiley \& Sons, 1981:218.

12. Landis JR, Koch GG. The measurements of observer agreement for categorical data. Biometrics 1977;33:159-74.

13. Sanders R. Intra-articular fractures of the calcaneus: present state of the art. J Orthop Trauma 1992;6:252-65. 